\title{
Taming Uncertainty: The Limits to Quantification
}

ARTICLE in ASTIN BULLETIN · JANUARY 2016

Impact Factor: 0.74 · DOI: 10.1017/asb.2015.29

READS

94

3 AUTHORS, INCLUDING:

\section{Andreas Tsanakas}

City University London

34 PUBLICATIONS 281 CITATIONS

SEE PROFILE 


\title{
Taming Uncertainty: The Limits to Quantification ${ }^{1}$
}

This version: April 2015

\author{
Andreas Tsanakas ${ }^{2} \quad$ M Bruce Beck $^{3} \quad$ Michael Thompson $^{4}$
}

Taming the beast of uncertainty has been the grand project to which actuaries have dedicated much of their energy and skill over at least the last 50 years - roughly the time since, in Hans Bühlmann's (1989) famous term, 'Actuaries of the Second Kind' emerged. The collective efforts of actuarial researchers and practitioners have been founded on two premises:

- $\quad$ First Premise: Uncertainty is malleable; responsive to quantification skills and management expertise. Yes, actuaries concede, models sometimes fail, as seen in the case of the 2007-9 financial crisis. But, the world is orderly enough, such that one need not despair: the answer lies in using better, more sophisticated models, which will provide a better approximation to the risk environment.

- Second Premise: We can neatly separate the quantification of uncertainty - by probabilistic and statistical tools - from the economically motivated (and often normative) principles according to which decisions are taken. Disagreements about the accuracy of quantitative models are distinct from disagreements on how decisions should be taken. Maximising the Return on Capital for a portfolio is one thing; deciding that such a measure of performance should, in fact, be used in portfolio management is quite another.

The big task of taming uncertainty is thus broken down into two smaller tasks: first, to develop a statistical/stochastic model according to the best science we have access to; then, to consider how that model is used to take decisions such as pricing liabilities, setting capital requirements or optimising portfolios.

Such a conventional separation also leads to a rather neat definition of model risk: the risk arising from feeding the outputs of a flawed model to the (presumed correct) decision principle, thus leading to poor decisions. "Flawed" here refers to problems of model mis-specification, as well as inappropriate use (Board of Governors of the Federal Reserve System, 2011). (In fact, these two sources of model risk are hardly distinct: since we have no access to some true model of nature, appropriateness of a model can only ever be understood with reference to specific uses and applications.) But if there is agreement on decision principles, implying that, equipped with some ideally correct model we could perfectly manage risk, then all remaining risk must be model risk there is nothing else. Thus, our concerns about the state of the world transmute into concerns

\footnotetext{
${ }^{1}$ To appear in ASTIN Bulletin, January 2016. We thank the Editor for helpful suggestions. This note draws on the report of the Institute and Faculty of Actuaries Working Party on Model Risk (2015), to which the authors contributed. We are grateful to members of the Working Party, as well as Andrew Hitchcox and Malcolm Kemp, for their invaluable feedback.

2 Faculty of Actuarial Science and Insurance, Cass Business School, City University London. a.tsanakas.1@city.ac.uk

${ }^{3}$ Department of Civil and Environmental Engineering, Imperial College London. mbbeck@uga.edu

${ }^{4}$ International Institute for Applied Systems Analysis. thompson@iiasa.ac.at
} 
about the state of our knowledge. The purported taming of uncertainty does not make risk disappear; it merely sublimates it into the epistemic domain.

Work under those two premises has led to a flourishing of the actuarial literature. One could talk in fact about a bifurcation along the lines discussed above. On the one hand, we have the great achievements in the domain of modelling, e.g. stochastic claims reserving, longevity models, and extreme value theory, while, on the other, we have the elegant contributions to decision making, e.g. risk measure theory, market consistent valuation, and optimal risk transfers. At the same time, we argue here, each of the two premises is flawed. In particular, the very conception of uncertainty, as encoded in those two premises, is too limited to reflect the complex financial world that actuaries have to navigate.

To start with, we need to talk about uncertainties, plural. We are all conscious of the distinction between risk, for which possible outcomes and probabilities are well specified and known, and uncertainty, where such knowledge is not given. The latter can itself be split into two kinds: on the one hand, epistemological uncertainty, reflecting difficulties in quantification due to the complexity of the problem at hand and the lack of sufficient data; on the other, ontological uncertainty, reflecting the possible existence of states and eventualities, of which decision makers are as yet unaware. Ontological uncertainty often arises from structural breaks, caused by changes in the environment (e.g. climate change) or radical innovation and changing market practices (Lane and Maxfield, 2005).

But there is also a fourth kind of uncertainty, generally not acknowledged in the actuarial or economics literatures, but studied comprehensively by anthropologists and political scientists ${ }^{5}$. Imagine a game of dice. Agents focused on risk will take the odds of different outcomes as given and focus on how to take optimal decisions. Those considering epistemological uncertainty will argue about the validity of the estimated odds (what if the six sides of a die are not equiprobable?), while those worried about ontological uncertainty will point to the limits of quantification (what if the dice are actually dodecahedra instead of cubes?). But there will also be some who articulate a very different objection to attempts at quantifying uncertainty: those who believe that the dice are fixed or that their counterparty is unlikely to pay up in any case ${ }^{6}$. For such agents, called cynics or fatalists by anthropologists, models are answering an irrelevant question; hence concern about their validity is misplaced. We call the uncertainty that this view represents, framing uncertainty, as it represents fundamental doubt about the 'rules of the game'. The rational response to such uncertainty is manipulation, blame-shedding and a focus on the short term.

\footnotetext{
${ }^{5}$ Perhaps its closest relatives in economics are Keynes' 'animal spirits', which are also a major theme in Greenspan's (2013) attempt to explain the financial crisis of 2007-9. The anthropological theory is exposed in Thompson et al. (1990); a range of applications is given in Verweij and Thompson (2006). For an insurance application see Ingram et al. (2012). To be precise, those works, dealing with plural rationalities, each endowed with its own Myth of Nature, are not overly concerned with risk models and thus do not engage in mapping such rationalities to types of uncertainty as is done here. To get to such a mapping, one has to somewhat switch perspective from conceptions of nature (or, rather, of simple representations of nature) to conceptions of complex computational models (as dealt with for environment-climate models in Beck (2014)). Such a switch of perspective is performed by the Working Party on Model Risk (2015).

${ }^{6}$ This attitude is captured by Leonard Cohen's song Everybody Knows: “Everybody knows that the dice are loaded / Everybody rolls with their fingers crossed / Everybody knows that the war is over / Everybody knows the good guys lost / Everybody knows the fight was fixed / The poor stay poor, the rich get rich"
} 
It is apparent that while risk and epistemological uncertainty are both malleable, that is, amenable to probabilistic modelling (though for the latter a Bayesian interpretation may be necessary), ontological and framing uncertainties do not lend themselves to such modelling. Hence they are not natural domains for the application of conventional actuarial skills.

A careful look at the role of risk models in the 2007-9 financial crisis reveals all those four kinds of uncertainty in play. It has been argued that the crisis revealed an intellectual failure of a monocultural economics and modelling, which focused exclusively on risk, ignoring epistemological and ontological uncertainties (Bronk, 2011). The use of Gaussian copulas in credit risk management is an instructive example (Donnelly and Embrechts, 2010): the emphasis on calculating default probability of a credit derivative like a CDO (risk) came at the cost of using models that were too simple, internally inconsistent, and did not capture tail dependencies appropriately (epistemological uncertainty). Such disregard of uncertainty was not because of naivety on behalf of modellers, who were perfectly aware of the limitations of the Gaussian copula model. Operational factors, such as the need for a clear way to book P\&L and thus set bonuses, provided the organisational context in which the Gaussian copula model became indispensable (MacKenzie and Spears, 2014).

Furthermore, the sensitivity of model outputs to assumptions that could in practice never be validated using historical data, given the context of a young market with complex products and transaction patterns, was not fully considered (ontological uncertainty). One could say: the problem was not that models were 'wrong', but that they could never be even close to 'right' (and that people chose to ignore this). Maybe most importantly, there was also a fundamental indifference to the accuracy of the models used (framing uncertainty), infamously captured by the comment of rating agency staff that a product "could be structured by cows and we would rate it" (Jones, 2008).

Returning to the First Premise, we would argue that the work of actuaries is rooted in the interplay between risk and epistemological uncertainty. Turning the latter into the former may have once been our aim: resolving uncertainty through quantification. The recent literature on model uncertainty is more sceptical about such aims, with explicit acknowledgment of statistical considerations (concerns of epistemological uncertainty) when formulating decision criteria (responses to risk), but still sees uncertainty in primarily technical terms; indicatively we mention Cairns (2000), Cont et al. (2010), Ziegel (2014) Bignozzi and Tsanakas (2015), Barrieu and Scandolo (2015) in the context of risk measurement. But ontological and framing uncertainties remain ignored. When actuaries ignore such uncertainties in their practice, it does not follow that they are oblivious to them. Rather, actuarial scientists are to varying extents conscious that paying too much attention to ontological and framing uncertainties would undermine the First Premise, and thus, the ability to impose enough mathematical structure on the world, such that modelling work can continue and lead to ever deeper understanding. Quantitative scientists may feel that they must downplay such uncertainties, in order to fulfil their professional and scientific identities.

This last point demonstrates another way in which anthropological theories of risk help us, beyond completing a classification of uncertainty types. The example of dice, while helpful as an illustration, is rather reductive, not least because it confines all considerations of human nature to one kind of uncertainty. Rather than attempting precise definitions of those uncertainty types, we find it more interesting to ask how diverse conceptions of uncertainty (and concern for those) are generated. They neither arise in a vacuum nor can they be reduced to the psychological profiles of individuals. Instead, alternative conceptions of uncertainty are closely bound up with ways of organising social 
and economic relations (Thompson et al., 1990). Thus, in the world of insurance risk management, focusing on reliably quantified risks allows speedy and efficient decision making. Acknowledging the prevalence of epistemological uncertainty necessitates more sophisticated quantification approaches and establishes the demand for highly qualified professionals ("people like us"). Concern for ontological uncertainties generates risk management that is both prudent and imaginative, employing scenario analyses, for example, and giving credence to the worries of emerging risk committees. Finally, framing uncertainty recognises that the game of insurance is often played at a different level, beyond the considerations of modellers.

If the ways in which we conceive and respond to uncertainty are at the same time causes and consequences of our differing ways of acting in the presence of uncertainty, then the Second Premise loses its plausibility: disagreements about the validity of statistical models and about the appropriateness of different decision mechanisms are not separable. When a decision maker applies pressure to a modeller to come up with figures that are "commercially meaningful", she is not challenging the technical validity of the model: she is merely asserting her own beliefs about what is a legitimate way of running an insurance business. When actuaries are asked by management to produce models that are accurate, detailed, and do not take days to run, this does not reflect naivety: it just manifests the need for decisions to be taken, today. And when a statistician vigorously protests against the practice of setting capital requirements using extreme percentiles, given the scarcity of relevant data (as is the case with Solvency II), he does not only make a statistical point: he is engaged in a battle to preserve his professional integrity.

A recent Working Party of the Institute and Faculty of Actuaries, to which the authors of this editorial contributed, was tasked to investigate the problem of model risk. In its report, the Working Party on Model Risk (2015) argued that four distinct conceptions of quantitative models and their legitimate use exist, which can be seen as responses to the four types of uncertainty discussed here. Furthermore, all such conceptions of models are necessary in risk management; at the same time, each is bound to lead to failure, if it becomes dominant within an organisation (see Tsanakas et al. (2014) for a brief summary). Focusing on risk and ignoring other forms of uncertainty leads to the illusion that uncertainty is tamed and to decision-makers' overconfidence. An excessive emphasis on epistemological uncertainty can lead to endless elaboration, while missing possible flaws in the paradigm itself that is required for such elaboration to take place. Concern for ontological uncertainty, while enabling 'thinking outside the box', can stifle innovation. Allowing the cynicism engendered by framing uncertainty to dominate, induces loss of accountability and blindness to useful evidence that models can provide.

In fact, managing (model) risk requires governance that explicitly acknowledges the presence of all four kinds of uncertainty and thus legitimises the diverse (non-)modelling practices of agents driven by conflicting conceptions of uncertainty and of quantitative models. Actuaries, while troubled by such relativism, have much to gain from it: investment in their models (in return for providing detailed management information); big-picture challenge (in return for accepting the limitations of their discipline); management accountability (in return for accepting commercial constraints).

So what sort of Actuary does this require - of the Fourth (D'Arcy, 2005) or maybe some Fifth Kind? We think this is partly a misplaced question. Of course actuaries must accept as legitimate the concerns of the different professionals with whom they work. But practitioners do not need us to 
tell them to find ways of aligning their interests with those of others within their organisations, in order to get their jobs done. Such awareness is nothing new and does not conflict with an actuarial professional identity. (Any instinctive democrat knows that it is not true that "hell is other people"; hell is too many people like us.) The challenge is primarily for institutions to foster and manage this diversity of uncertainty perceptions. In order to contribute to such diversity - and hold their own in the face of conflicting pressures that include the post-2008 backlash against mathematical risk models (prominently, Turner (2009)) - actuarial practitioners and researchers need to maintain a strong identity, based on technical excellence and professional integrity. This identity can only be further strengthened by exposure to a wider body of knowledge, including the anthropological approach to uncertainty discussed here.

\section{References}

Barrieu, P., and Scandolo, G. (2015). Assessing financial model risk. European Journal of Operational Research, 242(2), 546-556.

Bignozzi V., Tsanakas, A. (2014). Parameter uncertainty and residual estimation risk. Journal of Risk and Insurance, forthcoming.

Beck M. B. (2014). Handling Uncertainty in Environmental Models at the Science-policy-society Interfaces. In Error and Uncertainty in Scientific Practice (Boumans, M. Hon, G., and Petersen, A. C., eds), Pickering \& Chatto, pp. 97-135.

Board of Governors of the Federal Reserve System (2011). SR 11-7: Guidance on Model Risk Management. Available: http://www.federalreserve.gov/bankinforeg/srletters/sr1107a1.pdf

Bronk, R. (2011). Uncertainty, modelling monocultures and the financial crisis. Business Economist, $42(2), 5$.

BühImann, H. (1989). Actuaries of the Third Kind? ASTIN Bulletin, 19(S1), 5-6.

Cairns, A. J. (2000). A discussion of parameter and model uncertainty in insurance. Insurance: Mathematics and Economics, 27(3), 313-330.

Cont, R., Deguest, R., and Scandolo, G. (2010). Robustness and sensitivity analysis of risk measurement procedures. Quantitative Finance, 10(6), 593-606.

D'Arcy, S. (2005). On Becoming an Actuary of the Fourth Kind. Proceedings of the Casualty Actuarial Society (Presidential Address, November 14, 2005).

Donnelly, C., and Embrechts, P. (2010). The devil is in the tails: actuarial mathematics and the subprime mortgage crisis. ASTIN Bulletin, 40(01), 1-33.

Greenspan, A. (2013), The Map and the Territory. Risk, Human Nature and the Future of Forecasting. Penguin Press.

Ingram, D., Tayler, P., and Thompson, M. (2012). Surprise, surprise: from neoclassical economics to e-life. ASTIN Bulletin, 42(2), 389-411. 
Jones, S. (2008), Alphaville: Rating Cows. Financial Times, 23 October 2008.

Lane, D. A., and Maxfield, R. R. (2005). Ontological uncertainty and innovation. Journal of evolutionary economics, 15(1), 3-50.

MacKenzie, D., and Spears, T. (2014). 'A device for being able to book P\&L': The Organizational Embedding of the Gaussian Copula. Social Studies of Science, 44(3), 418-440.

Thompson, M., Ellis, R., and Wildavsky, A. (1990). Cultural theory. Westview Press.

Tsanakas A., Beck, M. B., Ford, T., Thompson, M., Ye, I. (2014). Model Risk and Culture. Actuary Magazine, December 2014.

Turner, A. (2009). The Turner Review: A regulatory response to the global banking crisis. Financial Services Authority; available: www.fsa.gov.uk/pubs/other/turner review.pdf

Verweij, M. and Thompson, M. (eds; 2006). Clumsy Solutions for a Complex World: Governance, Politics and Plural Perceptions. Palgrave Macmillan.

Working Party on Model Risk (2015). Model Risk: Daring to Open the Black Box. Presented to the Institute and Faculty of Actuaries on 23 March 2015. Available:

www.actuaries.org.uk/sites/all/files/documents/pdf/model-risk-working-party-paper.pdf

Ziegel, J. F. (2014). Coherence and elicitability. Mathematical Finance, forthcoming. 